\title{
Effects of Forebody Geometry on Side Forces on a Cylindrical Afterbody at High Angles of Attack
}

\author{
Timucin Serdaroglu ${ }^{1}$, Hamed Pahlavani ${ }^{2}$ and I Bedii Ozdemir ${ }^{2 *}$ \\ ${ }^{1}$ Systems Engineering, Turkish Aerospace Industries, Turkey \\ ${ }^{2}$ Faculty of Mechanical Engineering, Istanbul Technical University, Turkey
}

\begin{abstract}
The aim of the present study was to investigate the effects of the forebody geometry on the asymmetric side forces, separations and vortex shedding behind the vehicles at high angles of attack. It reports wall pressure measurements to describe the asymmetric vortex system developing over an inclined cylindrical body exposed to a low-speed freestream. The model with the tangent-ogive nose geometry of lengths $1.5 d, 2.5 d$ and $3.5 d$ was exposed to a uniform freestream with a mean velocity of $U_{\infty}=42 \mathrm{~m} / \mathrm{s}$ and at the angles of attack $\alpha=30,40,50$ and 60 degrees.

For the blunt and moderately streamlined forebody, the reattachment of the flow separated from the nose causes a local maximum on the lee side immediately after the forebody and introduces 3D effects on the boundary layer whose characteristics change from laminar to turbulent towards the aft of the body. As a result, the cross-flow boundary layer separation becomes asymmetric for the angles of attack equal or larger than 40 degrees. This leads to steady unbalanced side forces and significant yawing moment acting on the body. Depending on the forebody geometry, the unbalanced forces change its direction along the body and, when the angle of attack reaches 50 degrees, this happens twice.

The present results also indicate that when the angle of attack is increased to 60 degrees and beyond, the shape of the forebody has negligible effect on the nose separation because the separated flow no longer reattaches on the afterbody. Consequently, a turbulent cross-flow boundary layer separation occurs, which remains symmetric throughout the cylindrical body.
\end{abstract}

Keyword

Rocket aerodynamics, High angles of attack, Pressure measurements

\section{Introduction}

The target accuracy of missiles is a critical factor in their effectiveness. However, the intended target may not be often known prior to the launch and, therefore, retargetability nowadays has become an essential system attribute. Rapid delivery to the path from the launch point to the target demands an increased maneuverability, leading to flights at high angles of attack above 40 degrees [1]. Under these conditions, the flow around the vehicle is characterized by the presence of separations and vortex shedding $[2,3]$, which produce large asymmetric side forces $[4,5]$. The asymmetric flows at high angles of attack can be so prevailing that the control surfaces attached to the afterbody quickly becomes incapable of guiding the vehicle.

There have been many investigations concerning flows around the bodies of revolution, undertaken to understand the effect of the angle of attack on the complex flow features downstream of the forebody [6-11]. At low angles of attack ( $\alpha \leq 5$ degrees), the flow remains attached to the body except for the thickening of the boundary layer oc- curring on the lee side. For such flows, the lift force varies linearly with $\alpha$. As the angles of attack increases $(5 \leq \alpha \leq 25$ degrees), the flow encounters a positive transverse pressure gradient and develops a crossflow, which leads to the boundary layer separation on the lee side of the afterbody. Hence, the lift force varies nonlinearly. Furthermore, for blunted-nose bodies, a two-vortex system appears, first symmetric, but, as the angle of attack becomes large, the asymmetric vortices develop at the rear of the body without vortex shedding. As a result, the region, where the side force is effective, covers the entire afterbody. On stream-

*Corresponding author: I Bedii Ozdemir, Professor and Head of Fluids Group, Faculty of Mechanical Engineering, Istanbul Technical University, Gumussuyu 34437, Istanbul, Turkey

Accepted: January 13, 2020

Published online: January 15, 2020

Citation: Serdaroglu T, Pahlavani H, Ozdemir IB (2020) Effects of Forebody Geometry on Side Forces on a Cylindrical Afterbody at High Angles of Attack. J Aerosp Eng Mech 4(1):177-186 
lined bodies, however, the alternating vortex shedding occurs: At large $\alpha$ values, the shedding frequency increases and the swapping force cells become smaller. When the angles of attack increases to 60 degrees and beyond, the vortices on the lee side become always asymmetric in that several pairs of asymmetric vortices can form along the afterbody. The flow becomes disorganized and unsteady, which increases intermittent side forces. Depending on the flow component along the body, the complex structure can remain stable over time.

It seems that the vortex clusters appear to be the main source of the large unsteady side forces [12]. Therefore, it is essential to understand the changes in the state of the vortex system [13]. We know that any small perturbation emerging in the flow upstream is sufficient to transform the unstable symmetric vortex structure into a stable asymmetric state [14-16]. Zhu, et al. [17] applied micro-PIV to measure the flow near the tip and discovered that the asymmetric development of initial disturbances in shear layers leads to the downstream vortex asymmetry. Indeed, with the inviscid flow assumption [18-20], it has been numerically proven that the flow instabilities become the dominant mechanisms as the angle of attack increases and can lead to very large side forces. Furthermore, at high angles of attack, asymmetric vortex sheets can be encountered on the configurations where the separation lines are symmetric [9]. The contribution of viscosity on the changes of the vortex system is also of interest. At high angles of attack, it has been shown that when the Reynolds number becomes critical [21-23], while the separation remains laminar on the one side, a turbulent separation occurs on the other side of the afterbody [24]. As a result, the initially symmetric vortices degenerate into the asymmetric vortices [25]. However, the calculations [19] show that flows with such separations generate forces that are insignificant compared to large side forces observed in experiments. Yet, some other works (e.g. Lamont [2]) show that the side forces can be very large even at low Reynolds numbers.

It becomes clear that the flow instabilities are somehow responsible for the formation of stable asymmetric vortices, which eventually result in large side forces [26]. The upstream turbulence is one of the sources of flow irregularity, but only causes an asymmetric flow of random character [25]. It has been also known [13] that at high angles of attack, the side forces are not responsive to the direction of large upstream flow defects such as, asymmetric separation, etc. Other studies $[25,27]$ show that instabilities can even develop due to microscopic irregularities on almost perfect models. They are considered as the mechanisms responsible for the asymmetric vortex systems.

There appeared many works aiming to suppress or control the instabilities and to increase the maneuverability [28-34]. Hence, there is still a strong need to understand the basic mechanisms of the formation and persistence of the vortex systems. In the present work, an experimental approach has been employed to investigate the structure of asymmetrical vortex systems, developing on various forebody geometries and for different angles of attack. In particular, it is of interest how the vortex system evolves along the body after the tip perturbation $[35,36]$. The next section describes the model and the experimental techniques. Section 5 presents the results and discussions, and the paper ends with conclusions.

\section{Model Description and Experimental Proce- dures}

The experiments were conducted in an open circuit subsonic wind tunnel (Figure 1). Filtered air from the centrifugal fan expands through the inlet diffuser, which reduces the flow speed before the settling chamber where three mesh screens damp out flow irregularities and develop a low-turbulence uniform flow before the contraction. The contraction further reduces variations of the mean velocity and fluctuations to negligibly small values; the profile of the stream wise mean velocity at the inlet of the test section has a turbulent wall shear layer with a thickness (corresponding to the location at which the velocity reached $99 \%$ of the centerline value) $3 \%$ of the tunnel width. The test section was $500 \times 500 \mathrm{~mm}^{2}$ in cross-section and, to-

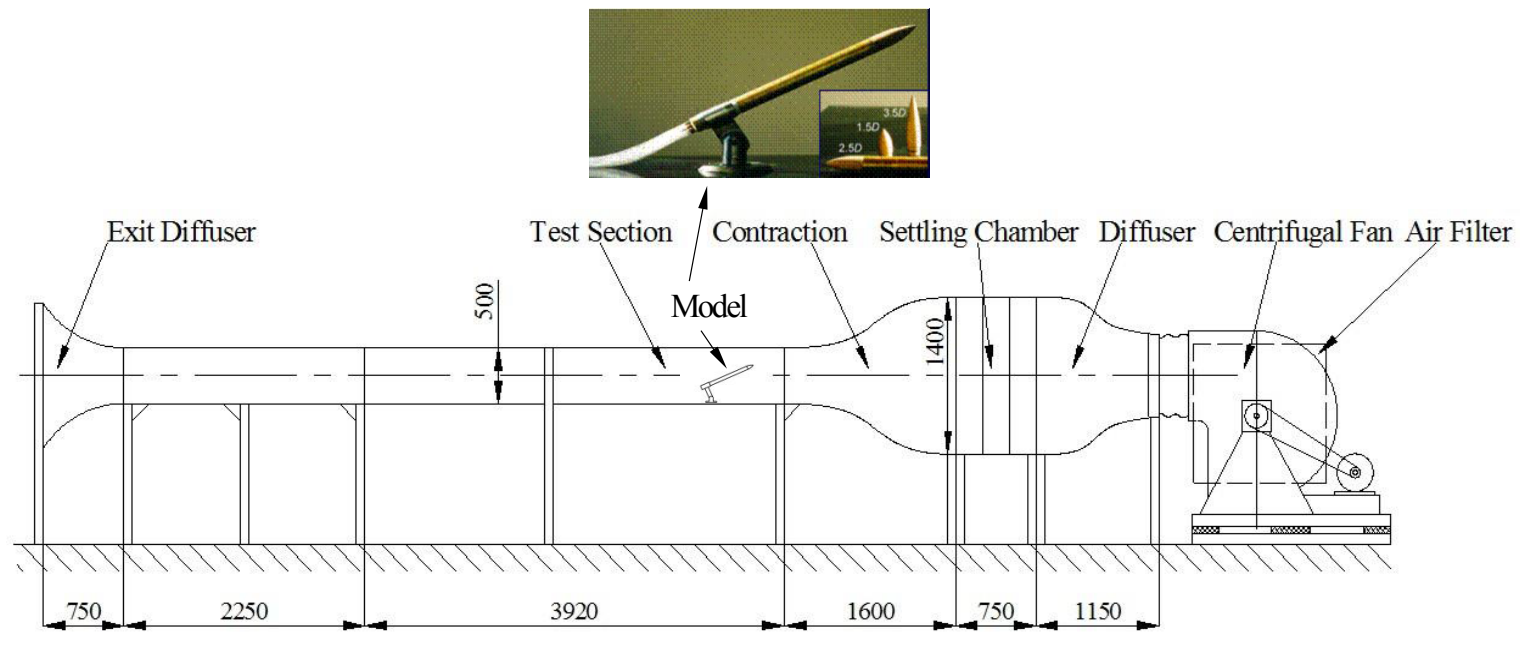

Figure 1: Schematic of the wind tunnel and the model (dimensions are in $\mathrm{mm}$ ). 
gether with the exit ducting, extends $5500 \mathrm{~mm}$ in length, with upper and side walls made of Plexiglas to allow light transmittance.

The model for the cylindrical afterbody (Figure 1 ) of diameter $d=30 \mathrm{~mm}$ and length $L=360 \mathrm{~mm}$ was manufactured from brass and the external surfaces were fine-finished. The forebody shape variations included tangent-ogive cones with a nose fineness of $0.5 \%$ and lengths $1.5 d, 2.5 d$ and $3.5 d$. A steel mounting pad of $60 \mathrm{~mm}$ height allowed the positioning of the model outside the boundary layers developing over the tunnel walls.

The edges of the strut were all streamlined and welded junctions were smoothly rounded to avoid any disturbance and flow separation under the model $[37,38]$. The model was exposed to a uniform freestream with a mean velocity of $U_{\infty}$ $=42 \mathrm{~m} / \mathrm{s}$ at angles of attack, $a=30,40,50$ and 60 degrees. These correspond to the Reynolds numbers $\left(\operatorname{Re} e_{\infty}=U_{\infty} d \operatorname{Sin}\right.$ $a / u$ ) from $0.4 \times 105$ to $0.7 \times 105$, which span the critical flow conditions where the slender bodies expose to the side forces [14]. The turbulence in the wind tunnel was sufficiently low with an intensity of $0.5 \%$, which was sufficiently low to avoid the repeatability problems $[13,14]$. We know that for the body shapes leading to separation (like the present model), the reattachment and related phenomena are particularly prone to the blockage effects. Therefore, the projected frontal area of the present model assembly was kept low for retaining an insignificant blockage ratio (less than $4 \%$ ) in the test section. Also, in order to avoid incorrect static pressure due to wake blockage at the tunnel collector, the model assembly was located $1000 \mathrm{~mm}$ downstream the entrance and $4500 \mathrm{~mm}$ upstream the exit of the test section. Hence, in the present experiments, the test section static pressure was $p_{0}=$ $82 \mathrm{~Pa}$ below the atmospheric pressure.

Starting from $25 \mathrm{~mm}$ downstream of the forebody, 14 holes (tapping stations) were drilled on the surface along a line parallel to the axis of the cylinder. The holes of 1 $\mathrm{mm}$ diameter were separated by $15 \mathrm{~mm}$ and, each had its own piping connected to the sensor. By rotating the model on its own axis, the surface pressure measurements were taken at every 10-degree roll angle, resulting in a pressure distribution over a total of 504 points around the model. Data acquisition, conversion, reduction and digital processing were all made with a signal-conditioning unit and an A/D board. The A/D board converted analogue signals into digital information with a resolution of 16 bits. 40,000 samples were acquired with a sampling frequency of $9 \mathrm{kHz}$ and, to satisfy Nyquist's criterion, the signals were lowpass filtered at $4 \mathrm{kHz}$. The pressure signal on the surface was transmitted with a flexible connection of length 500 $\mathrm{mm}$ and inner radius $2 \mathrm{~mm}$ to a miniature piezo resistive sensor. The possible resonance frequency of the tube and transducer system was estimated to be slightly exceeding $4 \mathrm{kHz}$ [39] which was proved to be well above the frequency content of the signals measured in this study. The uncertainty in pressure measurements was expected to be less than $1 \%$ over the most of the flow zone.
The time-averaged mean $(\bar{p})$ of the temporally stationary data on $N=40000$ discrete time points were rendered dimensionless by the dynamic pressure of the free stream. The pressure coefficient is defined as,

$$
C_{p}=\frac{\bar{p}-p_{0}}{0.5 \rho U_{\infty}^{2}}
$$

\section{Results and Discussion}

Figure 2 shows the mean pressure distributions at different cross-sections of the afterbody with the $1.5 d$ tangent-ogive nose. In that, the roll angles $\varphi=0$ or 360 degrees correspond to the upwind and $\varphi=180$ degrees to the downwind points of each cross-section. The left hand side (LHS) of the body with respect to the approaching flow is represented by $\varphi=0-180$ degrees and the right hand side (RHS) $\varphi=180$-360 degrees. The generic features of the flow for $\alpha=30$ degrees configuration will be discussed first at a location $x / d=2.3$. The changes towards the aft of the body $(x / d=8.8)$ will then be presented together with comparisons with the other $\alpha$ configurations.

For $\alpha=30$ degrees and $x / d=2.3$, the $C_{p}$ appears to have higher values (with a maximum of $C_{p, \max }=0.25$ ) in the frontal stagnation region, where the streamlines are diverted. Starting from the stagnation point ( $\varphi=0$ or 360 degrees), the flow accelerates in a laminar boundary layer, where the $C_{p}$ remains positive up to the roll angles $\varphi= \pm 40 \mathrm{de}$ grees (-40 degrees correspond 320 degrees). Then, the $C_{p}$ turns into negative values which decrease symmetrically on both sides of the stagnation point up to $\varphi= \pm 68$ degrees where a minimum of $C_{p, \max }=-0.5$ occurs. It levels out to a constant value at around $\varphi= \pm 85$ degrees until a second deep appears at around $\varphi= \pm 140$ degrees. Towards the aft of the body (with increasing $x / d$ ratio), we observe a shift in the $\varphi$-location of the $C_{p, \max }$ and, thus, the leveling $C_{p}$ out to a constant value (just after the $C_{p, \max }$ ) moves from $\varphi= \pm 85$ degrees at $x / d=2.3$ to $\varphi= \pm 110$ degrees at $x / d=6.3$. This process is also accompanied by a rise of the leveled value from $C_{p}=-0.4$ at $x / d=2.3$ to $C_{p}=-0.3$ at $x / d=6.3$. The results of the experiments on an inclined cylinder exposed to a cross-flow (Lamont, 1982) showed that the flow separation, defined by zero wall shear stress, corresponds to a leveling in the $C_{p}$ values. We can then conclude that towards the aft of the body, due to increase in velocity, the characteristics of the cross-flow boundary layer change from laminar to turbulent. As a result, while a laminar separation occurs immediately downstream of the forebody $(x / d=2.3)$, a turbulent separation develops particularly close to the aft region ( $x / d=6.3$ and onward). The maximum value of $C_{p, \max }$ at the stagnation point also changes along the body from 0.25 at $x / d=2.3$ to 0.4 at $x / d$ $=8.8$. As the angle of attack increases, we see that much larger values are obtained and $C_{p}$ reaches 0.75 for $\alpha=60$ degrees.

The features of wall pressure discussed so far look much like the flow around a cylinder placed normal to the approaching flow at a critical Reynolds number, $\operatorname{Re}=0.4 \times$ $10^{5}$ [40]. However, except for these similarities, the wall pressure here presents an additional local maximum on 
a)
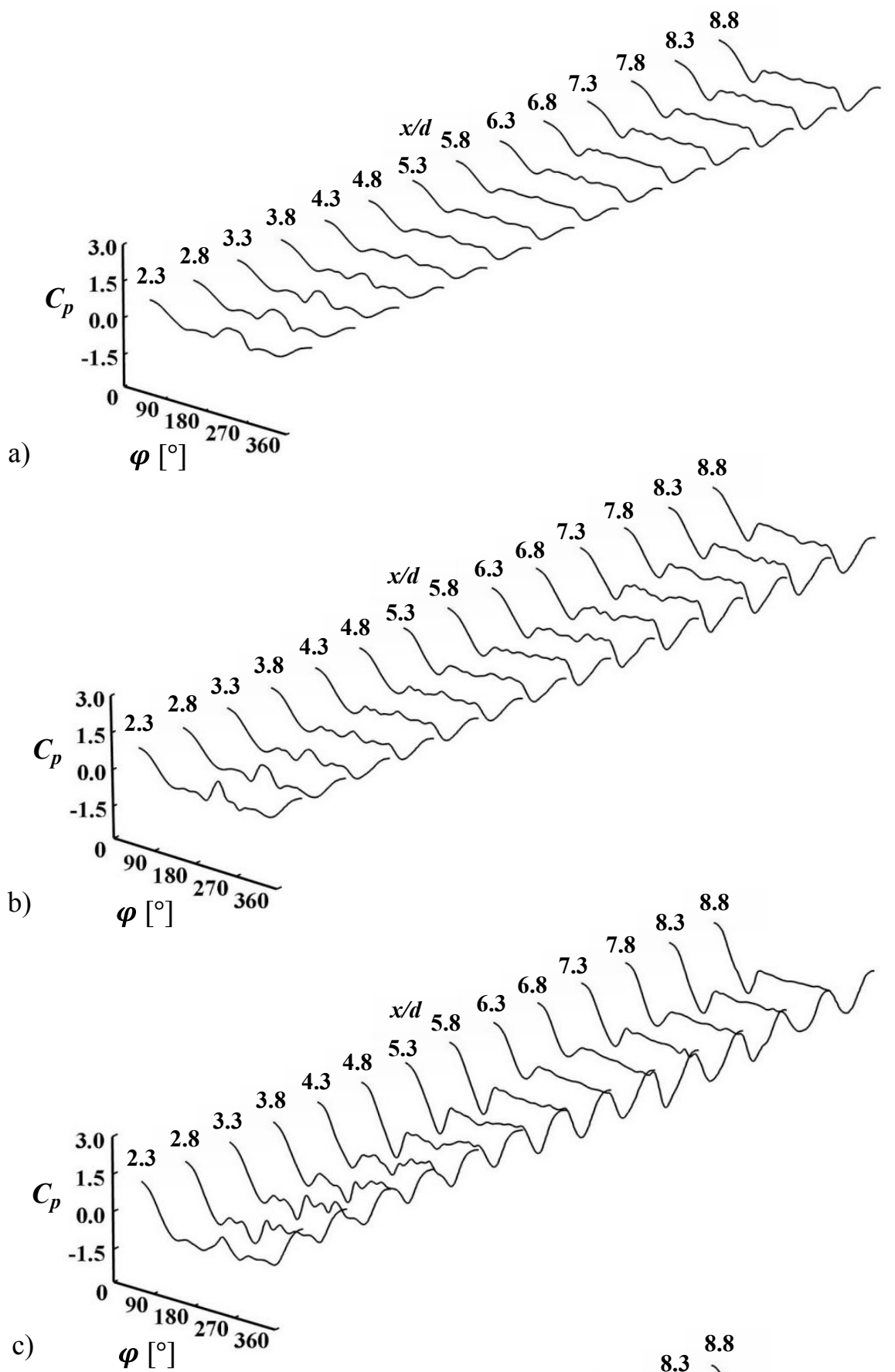

c)

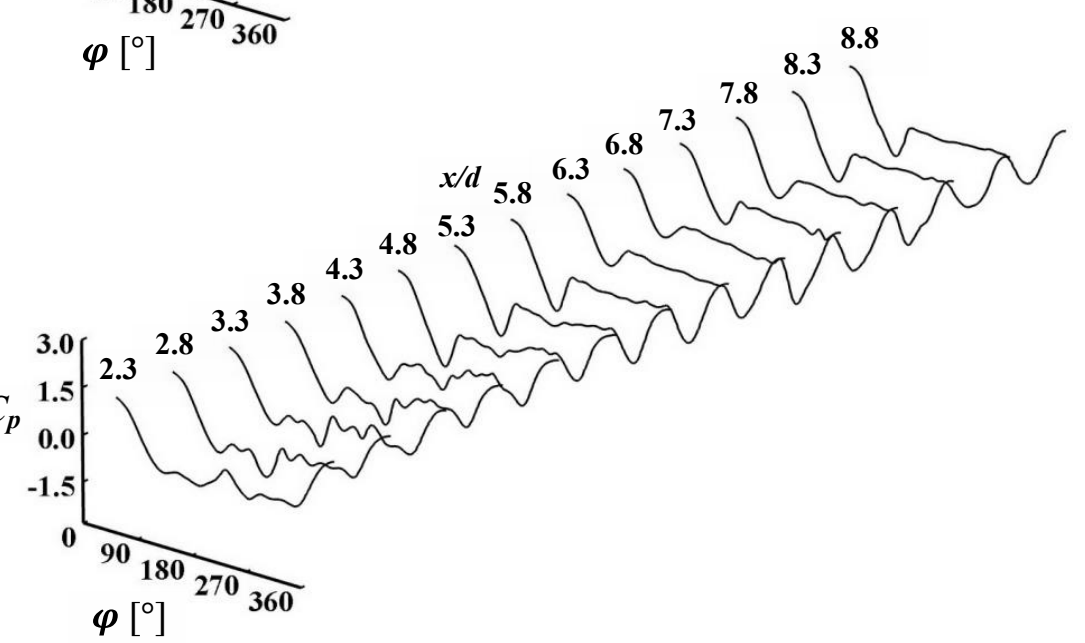

Figure 2: Distributions of pressure coefficient for $1.5 d$ tangent-ogive profile at a) $\alpha=30$, b) $\alpha=40$, c) $\alpha=50$ and d) $\alpha=60$ degrees. 
a)
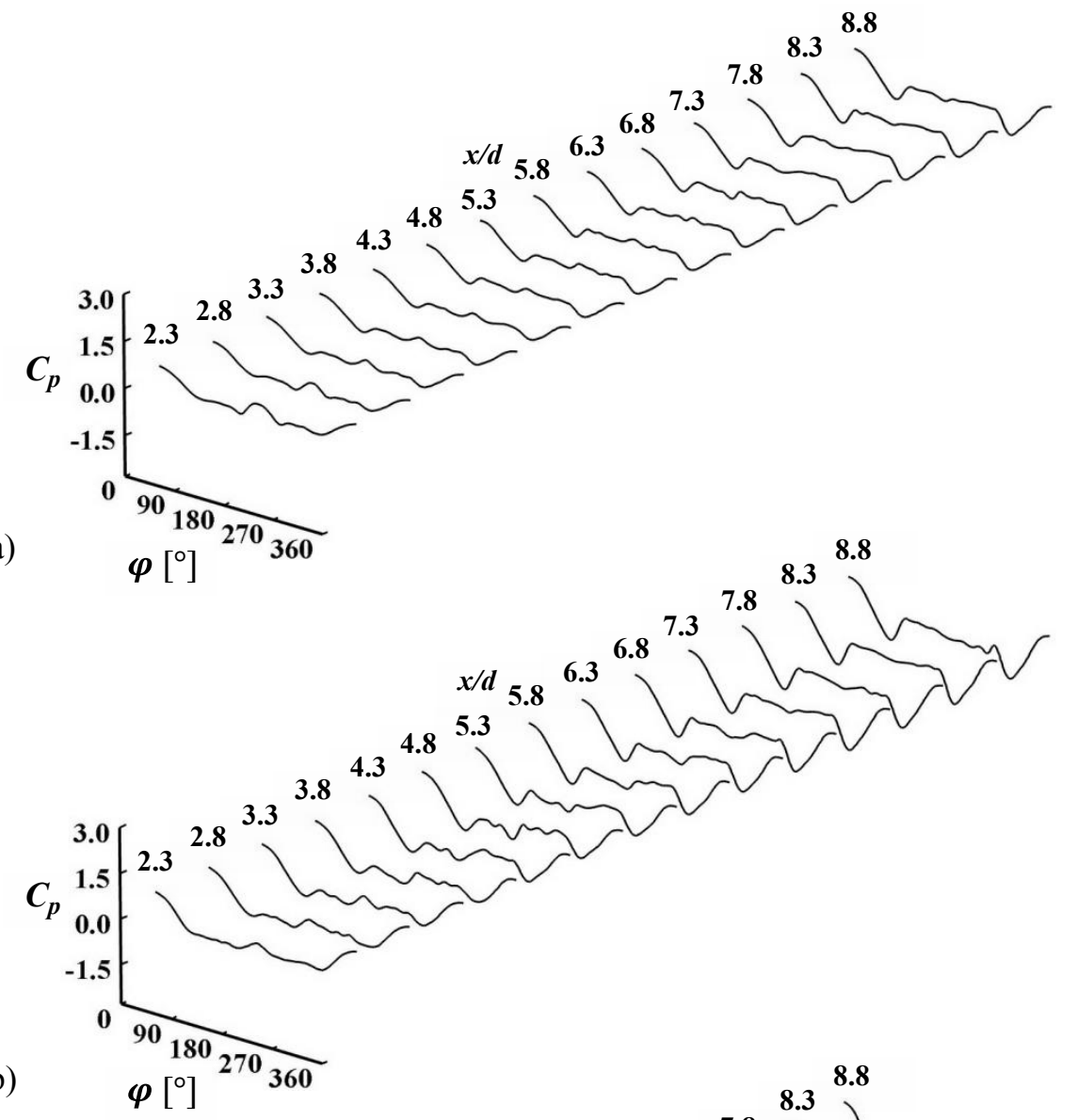

b)

c)
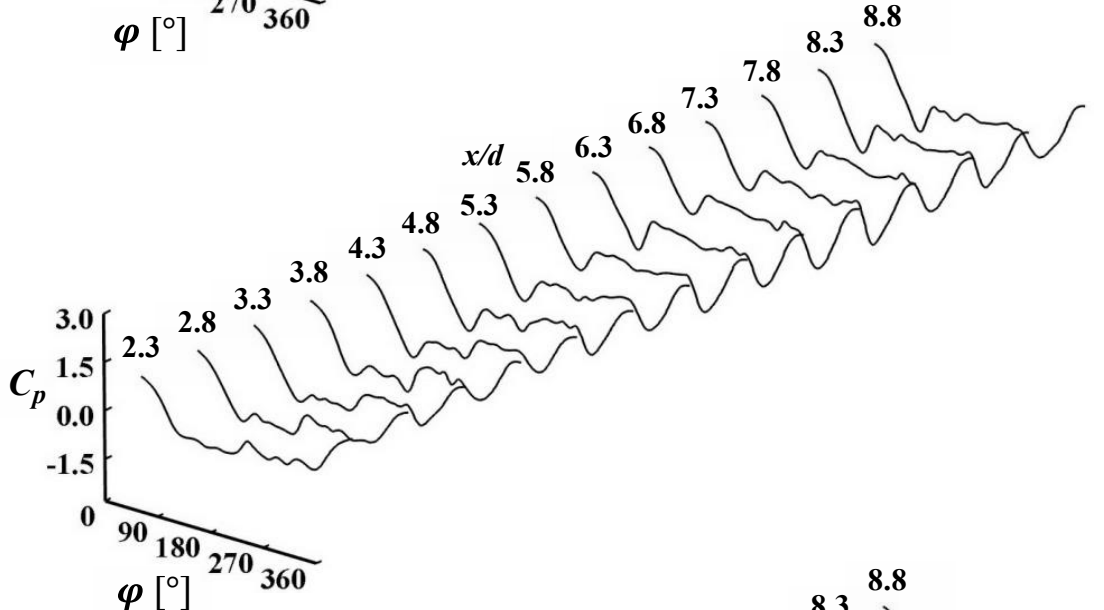

d)

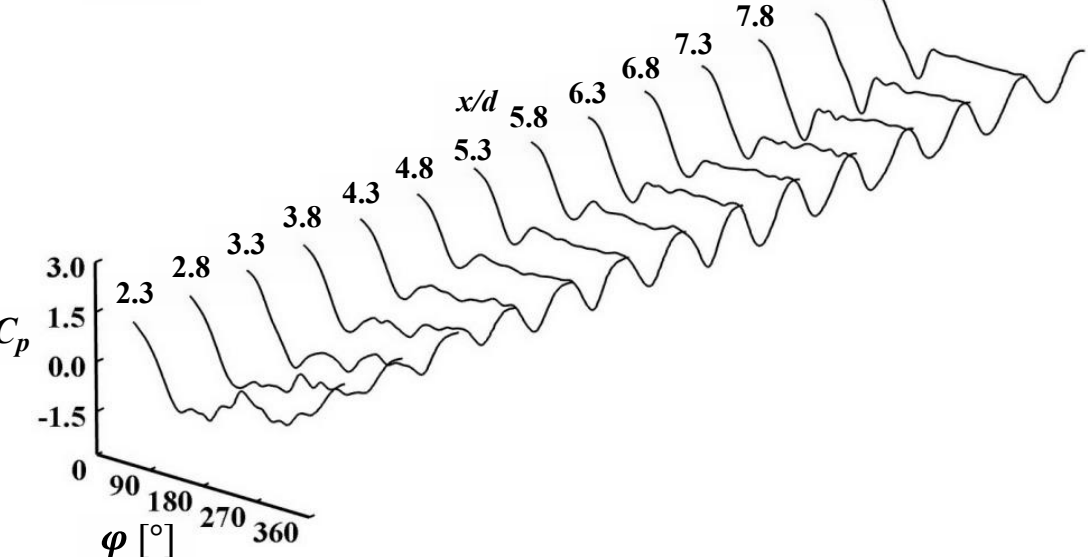

Figure 3: Distributions of pressure coefficient for $2.5 d$ tangent-ogive profile at a) $\alpha=30$, b) $\alpha=40$, c) $\alpha=50$ and d) $\alpha=60$ degrees. 
the lee side $\left(\varphi= \pm 180\right.$ degrees) where $C_{p}$ still has negative values. At $x / d=2.3$, the peak pressure on the lee side resides between the two minimums at $\varphi= \pm 140$ degrees and, as the minimums become closer (for example, $\varphi= \pm$ 160 degrees at $x / d=4.8$ ), the peak decreases and gradually disappears further along the body. The difference between the normal and inclined bodies exposed to the cross-flow stems from the fact that the former is a two-dimensional while the latter is a three-dimensional flow. Aside from the contribution due to the cross-flow separation occurring on a cylindrical body (positioned normal to the crossflow), for an inclined missile, the attachment of the flow separated from the nose imposes an extra impact on the lee side immediately after the forebody. The three-dimensionality of the flow induced by the separation from the nose is dominant just downstream of the forebody and diminishes further along the afterbody. Nonetheless, such flow detachments lead to nonlinear interactions and a possible vortex shedding [41], which introduces instability and some permanent jitter on the mean pressure on the lee side further along the body.

When $\alpha=40$ degrees, the footprints of the flow on the body surface qualitatively remain the same as $\alpha=30$ degrees, except for an increase in the difference between the profile minimum and maximum. Furthermore, the separation of the cross-flow boundary layer becomes asymmetric, in that it remains laminar (with $\varphi_{s} \leq 90$ degrees) on the left hand side of the body, whereas it looks turbulent (with $\varphi_{s}$ $\geq 110$ degrees) on the right hand side. As a result, the symmetry of the mean pressure profile observed immediately after the forebody deteriorates starting from $x / d=3.3$, and an asymmetry develops on the lee side where an increase in the $C_{p}$ values observed from the left to the right hand side. The asymmetry continues until $x / d=8.3$, where, as the separations become turbulent on both sides, the larger values on the right hand side are balanced by equal values on the left. The present results agree well with the findings of Zhongyang, et al. [32] for the blunted-nose forebody, which introduces a significant tip perturbation, interacting nonlinearly with the cross-flow boundary layer separation $[42,43]$. It is interesting to note that when the angle of attack, $\alpha$, is increased to 50 degrees, immediately after the disappearance of the peak on the lee side, a much stronger asymmetry appears with a sharp gradient of the mean wall pressure from the left to the right. More importantly, the asymmetry changes its direction along the body: While the larger mean pressures are prevailing on the right hand side up to $x / d=6.3$, in the aft region of the afterbody, the pressure becomes larger on the left hand side. Such steady unbalanced side forces are apparently expected to cause a significant yawing moment. As the angle of attack is further increased to $\alpha=60$ degrees, the turning point of the mean pressure asymmetry shifts up in the forward direction to $x / d=5.8$.

Figure 3 presents the mean pressure distributions on the body with the $2.5 d$ tangent-ogive nose profile. For $\alpha$ $=30$ degrees, it is clear that by streamlining the forebody, first the degree of three-dimensionality and then the re- sulting higher pressures on the lee side are reduced in amplitude. They diminish much earlier along the afterbody than the case with the $1.5 \mathrm{~d}$ blunt nose. However, the asymmetry developing when the angle of attack increased to 40 degrees still prevails with the larger $C_{p}$ values on the right hand side of the mean pressure profile. The change in the direction of asymmetry observed for $\alpha=50$ degrees in the blunt nose $(1.5 d)$ still exists but, in the streamlined $(2.5 d)$ nose profile, the gradients become milder and the turning point of the mean pressure asymmetry moves in the aft direction (to $x / d=6.8$ ). When $\alpha=60$ degrees, there is almost no difference in the characteristics of the mean pressure distribution for both the blunt and streamlined forebody. This shows that when the angle of attack is increased beyond 50 degrees, the shape of the forebody has negligible effect on the nose separation and the flow around the afterbody. This happens because, for these cases, the separated flow is no longer reattaches on the body.

When the forebody geometry has the $3.5 d$ tangent-ogive nose profile, Figure 4 , the flow over the cone remain attached for $\alpha=30$ degrees and the three-dimensional flow effects nearly vanish. A turbulent cross-flow boundary layer separation occurs with $\varphi_{s} \geq 110$ degrees and the flow remains symmetric throughout the afterbody. Since there would be no steady mean pressure asymmetry, one cannot expect a steady yawing moment. For $\alpha=40$ degrees, although the cross-flow boundary layer separation is turbulent both on the left and the right hand side of the body, the mean pressure increases first from the left to the right hand side up to $x / d=7.8$ and, then, from the right to the left hand side of the afterbody. The gradients of unbalanced mean pressures are milder, but a steady yawing moment still prevails. When $\alpha$ is increased 50 degrees, an interesting phenomenon is observed: The asymmetry occurs first in the direction with larger $C_{p}$ values on the right hand side, which lasts up to $x / d=6.3$, then on the left hand side up to $x / d=9.3$ and, finally, on the right hand side again in the aft region. In between the switching cells of unbalanced side forces along the body, there are two turning points of the mean pressure asymmetry. This means that the micro-jet blowing schemes [34], used for controlling the patterns of asymmetric flow to adjust the direction and magnitude of the unbalanced side-forces, need to be adjusted in multiple injections made at several stations along the afterbody. Indeed, this is in agreement with the fact that the control strategy with the jet blowing must depend on the $x / d$-location along the afterbody $[32,33]$. On the other hand, the measurements by Gao, et al. [29] suggest that the blowing passive jets as an effective control approach only works at the low blowing angles and, indeed, has no significant use in controlling the steady unbalanced side forces. Apart from mean flow characteristics, it is clear that if the system of unbalanced forces fluctuates, it will lead to fluctuating yawing moment, making the missile control nearly impossible. Since the jet blowing reduces the wake width and the vortex formation length [29], it may actually become effective particularly in suppressing the fluctuating forces. 


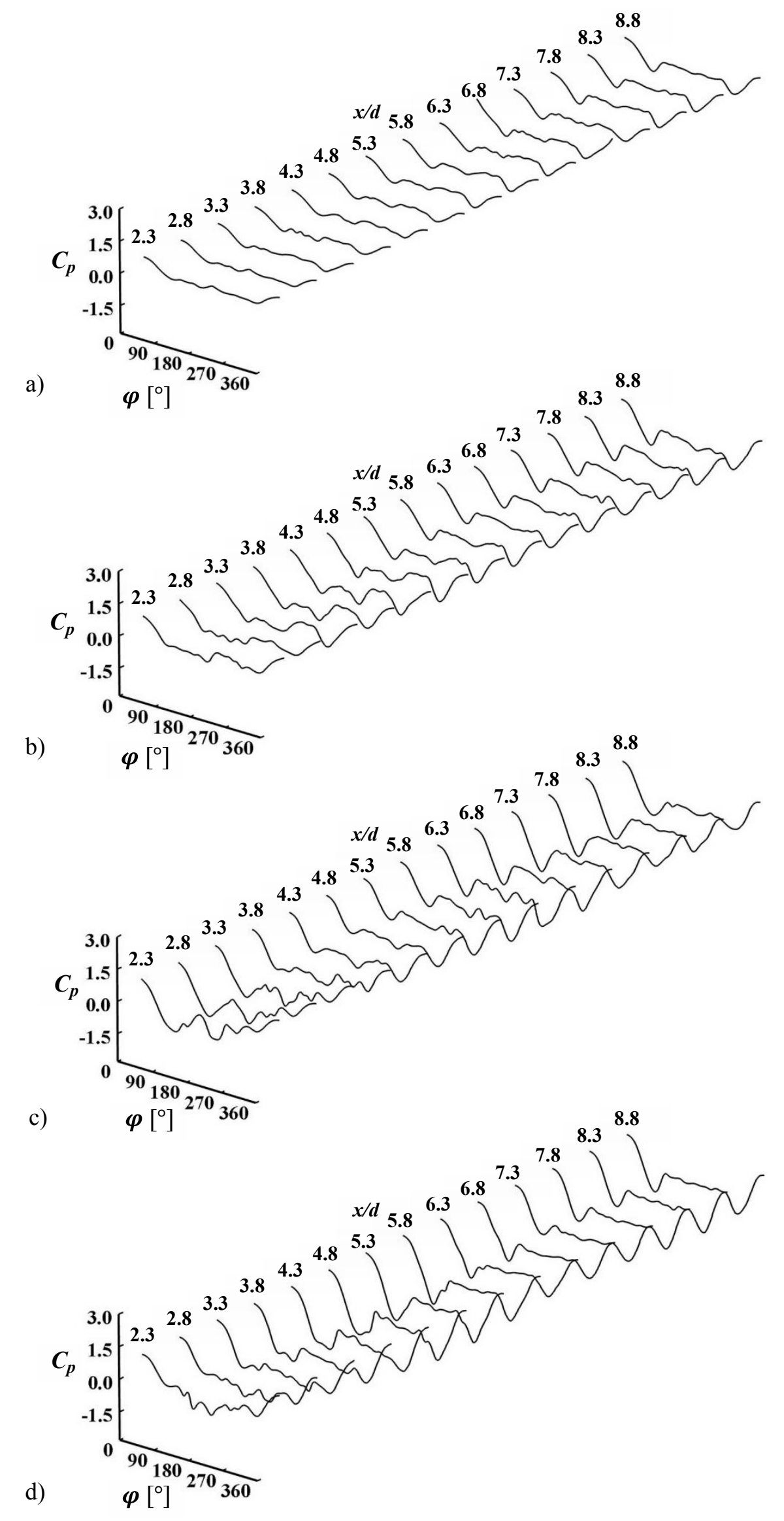

Figure 4: Distributions of pressure coefficient for 3.5d tangent-ogive profile at a) $\alpha=30$, b) $\alpha=40$, c) $\alpha=50$ and d) $\alpha=60$ degrees. 

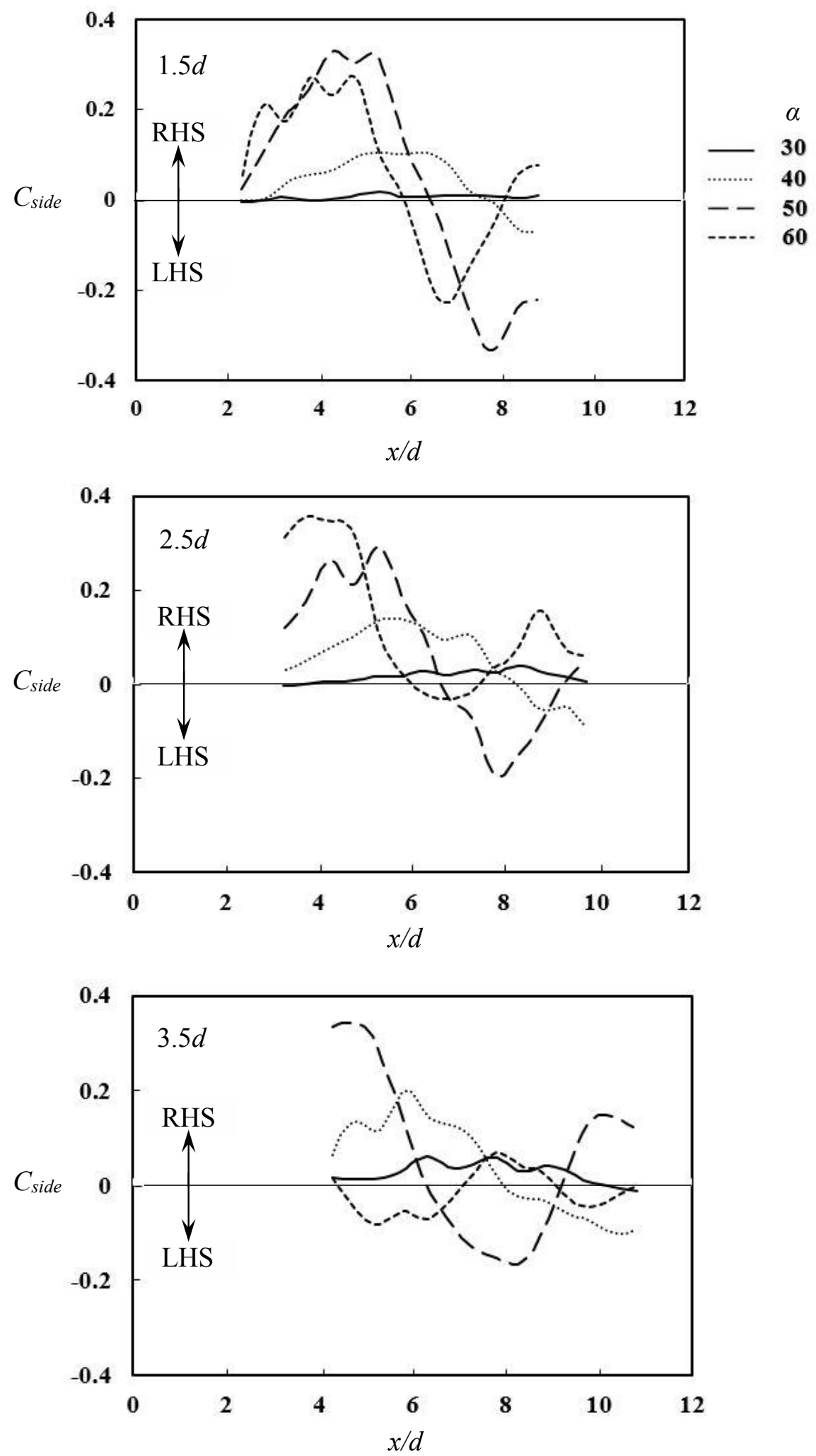

Figure 5: Side force coefficient for different tangent-ogive profiles.

When $\alpha$ is further increased to 60 degrees, there is no apparent asymmetry of the mean pressure profile. It can be concluded that when the flow separates from the nose without reattachment, except for the immediate downstream of the forebody, the cross-flow boundary layer becomes more stable and symmetric.
Figure 5 shows the variation of the side force along the forebody, which was calculated by integrating the pressure coefficients. It seems that the body with the $1.5 \mathrm{~d}$ tangent-ogive nose profile was not exposed to any significant asymmetric side force when the angle of attack remains less or equal to 30 degrees. As the angle of attack increas- 
es, the asymmetry first appears at $\alpha=40$ degrees, where a net side force acts on the right hand side $(\varphi=180-360$ degrees) of the body which remains one-sided except for a small aft region. Thus, this configuration is not expected to induce a significant yawing moment. A further increase in the angle of attack develops a strong asymmetry in the distribution of the side forces. The direction of the asymmetry changes sharply along the body, in that the side forces first act on the RHS surfaces of the cylindrical body (pushing it from right to left) and then, after $x / d=6.3$, are applied on the LHS surfaces (pushing the body from left to right). The largest yawing moment is expected to occur when $\alpha=50$ degrees, which sharply decreases when $\alpha$ reaches 60 degrees. In the latter case, the distribution of the side force along the body changes its direction of asymmetry once again back to the RHS surfaces.

For the $2.5 d$ tangent-ogive nose profile, the magnitudes of asymmetric side forces increase for small angles: It remains relatively small and right-sided for $\alpha=30$ degrees. For $\alpha=40$ degrees, the direction of the asymmetry changes from the right-sided one to the left-sided one. This means that for the $2.5 d$ tangent-ogive nose, the yawing moment develops at slightly lower angles of attack compared to $1.5 d$ tangent-ogive nose. This is also confirmed by Kaushik and Bala [44] who emphasized that at high forebody slenderness ratios, the change in the side force coefficient becomes very sharp beyond $\alpha=40$ degrees. For $\alpha=50$ degrees, the form of the side force distribution remains nearly the same except for a slight decrease in magnitudes. When $\alpha$ increases to 60 degrees, apart from a small region between $x / d=6.3$ and 7.8 , the asymmetry becomes nearly one-sided and is dominated by small values.

For the 3.5d tangent-ogive nose profile, the side force distributions appear asymmetric at all angles of attack. Although the asymmetry remains one-sided (acting on the RHS) at $\alpha=$ 30 degrees, it changes its direction along the body at higher angles of attack. Hence, strong yawing moments are expected to occur at large angles of attack, of which the most prominent is at $\alpha=50$ degrees.

\section{Conclusions}

The wall pressure measurements were used to study the effects of the forebody geometry on the asymmetric vortex system emerging on a cylindrical afterbody exposed to a lowspeed freestream at different angles of attack. The results can be highlighted as follows:

The separation of the cross-flow boundary layer becomes asymmetric when the angle of attack reaches 40 degrees, and any further increase in the angle (up to 50 degrees) leads to a phenomenon where the asymmetry changes its direction along the afterbody.

It is interesting that the streamlining of the forebody geometry seems to have strong effects on this feature: For the $1.5 d$ tangent-ogive nose profile, the change in the direction of asymmetry occurs only once with a single turning point, but as the streamlining of the forebody geome- try increases, the asymmetry changes its direction twice with two turning points.

It is also of note that when the angle of attack is increased to the 60 degrees and beyond, the shape of the forebody has almost negligible effect on the flow and the pressure distribution on the rear surfaces of the afterbody. This happens because, for these angles, the separated flow no longer reattaches on the afterbody.

\section{Acknowledgments}

This work has been supported by TUBITAK (MISAG 98). Partial support by TUBITAK SAGE is greatly acknowledged.

\section{References}

1. Champigny $P$ (1994) High Angle of Attack Aerodynamics. AGARD $\mathrm{CP}$ special course on missile aerodynamics, Specialised Printing Services Limited, Loughton, England.

2. Lamont PJ (1982) The complex asymmetric flow over a 3.5D ogive nose and cylindrical afterbody at high angles of attack. AIAA Paper.

3. Ericsson LE, Reding JP (1986) Asymmetric vortex shedding from bodies of revolution, In: MJ Hemsch, JN Nielsen, Tactical Missile Aerodynamics, Progress in Astronautics and Aeronautics Series, 243-296.

4. Wardlaw AB, Morrison AM (1976) Induced side forces at high angle of attack. Journal of Spacecraft and Rockets 13: 10.

5. Ericsson LE, Reding JP (1980) Vortex-induced asymmetric loads in 2D and 3D flows. AIAA Paper.

6. Letko W (1953) A low-speed experimental study of the directional characteristic a sharp nosed fuselage through a large angle of attack range at zero angle of sideslip. NACA-TN-2911.

7. Pick GS (1971) Investigation of side forces on ogive-cylinder bodies at high angles of attack in the M equals 0.5 to 1.1 range. AIAA Paper.

8. Keener ER, Chapman GT (1977) Similarity in vortex asymmetries over slender bodies and wings. AIAA Journal 15: 9.

9. Degani D, Levy Y (1992) Asymmetric turbulent vortical flows over slender bodies. AIAA Journals 30: 9.

10. Nelson RC, Malcolm GN (1992) Visualization of high angle of attack flow phenomena. In: Michael J Hemsch, Jack N Nielsen, Tacticle Missile Aerodynamics. Progress in Astronautics and Aeronautics Series 141.

11. Abney EJ, McDaniel MA (2005) High angle of attack aerodynamic predictions using missile datcom. AIAA paper.

12. Deng XY, Tian $\mathrm{W}, \mathrm{Ma} B F$, et al. (2008) Recent progress on the study of asymmetric vortex flow over slender bodies. Acta Mech Sin 24: 475-487.

13. Malcolm GN (1991) Forebody vortex control. Progress in Aerospace Sciences 28: 171-234.

14. Keener ER, Chapman GT, Kruse RL (1976) Effects of Mach number and afterbody length on onset of asymmetric forces on bodies at zero sideslip and high angles of attack. AIAA Paper.

15. Dexter PC, Hunt BL (1981) The effect of roll angle on the flow over a slender body of revolution at high angles of attack. AIAA paper.

16. Moir IRM (1990) An experimental investigation of the effect of 
fineness ratio on lateral forces on a pointed body of revolution. Vortex Flow Dynamics. 19-1-19-3.

17. Zhu Y, Yuan H, Lee C (2015) Experimental investigations of the initial growth of flow asymmetries over a slender body of revolution at high angles of attack. Phys Fluids 27.

18. Smith AC (1973) On the stability of Föppl's vortices. J Appl Mech 40: $610-612$.

19. Fiddes SP, Williams AL (1989) Recent developments in the study of separated flow past slender bodies at incidence. Symposium on the Prediction and Exploitation of Separated Flow 31.1-31.17.

20. Cai J, Liu F, Luo S (2003) Stability of symmetric vortices in two dimensions and over three-dimensional slender conical bodies. $J$ Fluid Mech 480: 65-94.

21. Champigny P (1984) Reynolds number effect on the aerodynamic characteristics of an ogive-cylinder at high angles of attack. AIAA Paper.

22. Lamont PJ (1985) The effect of Reynolds number on normal and side forces on ogive-cylinders at high incidence. AIAA Paper.

23. Schewe G (2001) Reynolds-number effects in flow around moreor-less bluff bodies. J Wind Eng Ind Aerod 89: 1267-1289.

24. Lamont PJ (1980) Pressure measurements on an ogive-cylinder at high angles of attack with laminar, transitional or turbulent separation. AIAA Paper.

25. Hunt BL, Dexter PC (1979) Pressures on a slender body at high angle of attack in a very low turbulence level air stream. AGARD CP 247-17.

26. Champigny $P$ (1986) Stability of side forces on bodies at high angle of attack. AIAA paper.

27. Dexter PC (1984) A study of asymmetric flow over slender bodies at high angles of attack in a low turbulence environment. AIAA paper.

28. Zhang W, Liu X, Zhai J, et al. (2012) Experimental study on side force alleviation of conical forebody with a fluttering flag. Phys Fluids 24.

29. Gao DL, Chen WL, Li H, et al. (2017) Flow around a slotted circular cylinder at various angles of attack. Exp Fluids 58: 132.

30. Wang QT, Cheng KM, Gu YS, et al. (2018) Continuous control of asymmetric forebody vortices in a bi-stable state. Phys Fluids 30.
31. Meng XS, Long YX, Wang JL, et al. (2018) Dynamics and control of the vortex flow behind a slender conical forebody by a pair of plasma actuators. Phys Fluids 30.

32. Qi ZY, Wang YK, Wang L, et al. (2017) Investigation on asymmetric flow over a blunt-nose slender body at high angle of attack. Fluid Dyn Res 49.

33. Qi ZY, Zong SY, Wang YK, et al. (2018) Sources of asymmetric flow over the blunt-nose slender body. Eur J Mech./B Fluids 75: 372-381.

34. Qi ZY, Wang YK (2019) Control of asymmetric flow over a bluntnosed slender body. Phys Fluids 31

35. Ma BF, Huang $Y$, Deng XY (2016) Dynamic responses of asymmetric vortices over slender bodies to a rotating tip perturbation. Exp Fluids 57.

36. Sha YX, Wang YK, Qi ZY (2017) Effect of nose perturbation on asymmetric vortices over a blunt-nose body at high angle of attack. Journal of Physics: Conf Series 822.

37. Mokry M (1995) Wall interference correction to drag measurements in automotive wind tunnels. J Wind Eng Ind Aerod 56: 107-122.

38. Cigada A, Falco M, Zasso A (2001) Development of new systems to measure the aerodynamic forces on section models in wind tunnel testing. J Wind Eng Ind Aerod 89: 725-746.

39. Bergh $\mathrm{H}$, Tijdeman $\mathrm{H}$ (1965) Theoretical and experimental results for the dynamic response of pressure measuring systems. NLR report.

40. Schlichting H, Gersten K (2000) Boundary-Layer Theory, 8th revised and enlarged edition. Spinger, Germany.

41. Ma BF, Liu TX (2014) Low-frequency vortex oscillation around slender bodies at high angles-of-attack. Phys Fluids 26.

42. Fagley C, Farnsworth J, Porter C, et al. (2013) Open-loop dynamics of the asymmetric vortex wake behind a von Kármán ogive at high incidence. Int J Flow Cont 5: 59-78.

43. Ma BF, Deng XY, Rong Z, et al. (2015) The self-excited rolling oscillations induced by fore-body vortices. Aerosp Sci Technol 47: 299-313.

44. Kaushik V, Bala S (2016) Investigation of side forces on conical cylindrical body at subsonic speeds. IJIET 7: 328-331.

DOI: $10.36959 / 422 / 439$

Copyright: (C) 2020 Serdaroglu T, et al. This is an open-access article distributed under the terms of the Creative Commons Attribution License, which permits unrestricted use, distribution, and reproduction in any medium, provided the original author and source are credited. 